\title{
Strategies and guidance for establishing a practical radiation protection culture in Europe in case of long term radioactive contamination after a nuclear accident: The SAGE Project
}

\author{
S. Lepicard ${ }^{1}$, I. Fiedler ${ }^{2}$, V. Nesterenko ${ }^{3}$, A. Nisbet ${ }^{4}$ and A. Sudas ${ }^{5}$ \\ ${ }^{1}$ Centre d'Étude sur l'Évaluation de la Protection dans le Domaine Nucléaire (CEPN), \\ Route du Panorama, BP. 48, 92263 Fontenay-aux-Roses Cedex, France \\ e-mail: lepicard@cepn.asso.fr \\ ${ }^{2}$ Forschunzszentrum für Umwelt und Gesundheit (GSF), Germany \\ ${ }^{3}$ The Belarusian Institute of Radiation Safety (BELRAD), Belarus \\ ${ }^{4}$ National Radiological Protection Board (NRPB), UK \\ ${ }^{5}$ Brest Branch of the Research Institute of Radiology (BB RIR), Belarus
}

\begin{abstract}
The evaluation of the rehabilitation strategies implemented in the contaminated territories of the CIS countries affected by the accident at the Chernobyl Nuclear Power Plant highlighted the need and importance to involve the population in the day-to-day management of the radiological situation, to complement the rehabilitation programme implemented by public authorities. The ETHOS experience in Belarus has revealed that to be effective and sustainable, this involvement must rely on the dissemination of a practical radiological protection culture within all sectors of the population and especially within professionals in charge of public health. The objective of the SAGE Project is to contribute to the development of strategies and guidance for implementing and disseminating such a culture in Western Europe, in case of a nuclear incident or accident with long-term radiological consequences. This paper presents the structure of the project, the stakeholder involvement process which has driven its realisation and the handbook on practical radiation protection culture which was elaborated, with different associated computer tools for people living in a contaminated territory.
\end{abstract}

\section{INTRODUCTION}

The evaluation of the rehabilitation strategies implemented in the contaminated territories of the CIS countries affected by the Chernobyl catastrophe pointed out the need and importance to involve the population in the day-to-day management of the radiological situation. This inclusion of local stakeholders complements the rehabilitation programme implemented by the public authorities, rather than replacing it.

The ETHOS experience in Belarus has revealed that to be effective and sustainable, this involvement of local stakeholders must rely on the dissemination of a practical radiological protection culture within all sectors of the population, and primarily with professionals in charge of public health [1-2]. ETHOS findings have provided a concrete basis for the development of such a practical culture in the specific context of Belarus. This has involved the combining of several factors:

- Direct access to radiological monitoring equipment or data, by which the radiological quality of the environment can be evaluated and the levels of internal and external exposure of individuals and the whole population can be controlled, 
- Tools that facilitate the understanding and the practical use of information related to the radiological situation (measurements, exposure routes, comparison data, improvement options) to be shared by the population and the health professionals and the measurement professionals.

The adoption of such a "practical radiological protection culture" for people living in a long-term contaminated territory contributes to the maintenance of both individual and collective vigilance relating to radiological protection issues. This favours active participation of concerned stakeholders in the protection of themselves and of the future generations. The emphasis placed on the interaction between individual and collective actions is crucial to take account of expectations and responsibilities of all those concerned. As demonstrated later, this interaction can be ensured by providing specific places for dialogue between the different stakeholders, and the use of specific tools for aiding information exchange about the problems posed by the long-term presence of radioactivity in the environment [3].

Following the ETHOS international seminar held in November 2001 in Stolyn (Belarus) [4], a declaration was signed by Belarusian national representatives and by different international organisations, stating that such an approach was "efficient in practice" and "merited to be studied, developed and disseminated" [5].

The SAGE Project supported by the European Commission [6], during the period October 2002 and March 2005, will develop strategies and guidance for implementing and disseminating a practical radiological protection culture in Western Europe, in case of a nuclear incident or accident with long-term radiological consequences. The eventual goal will be to have a system in place that could be applied throughout the European Union, but as a first step the proposed strategies and guidance will be elaborated on the basis of the three Member States involved in the Project - France, Germany and the United Kingdom.

\section{THE SAGE PARTNERSHIP AND WORK PROGRAM}

The partnership of the Project is composed of five teams from the following institutions: BB RIR (Belarus), BELRAD (Belarus), CEPN (France), GSF (Germany) and NRPB (United Kingdom). The expertise of this work group covers mainstream disciplines such as radiation protection (including public health issues), dose and risk assessment, radiation monitoring (including measurements of foodstuffs and body contamination), radioecology, environmental monitoring, economics, social management of risk, countermeasures after accidents, regulation and risk policies.

The SAGE work program is split into five work packages which encompass two major tasks:

- A "classical” review performed by the Project partners, of the existing regulatory and radiological monitoring infrastructures dedicated to long-term management of the contamination of the environment, in the three Member States involved as well as in Belarus, including the feedback from the ETHOS experience,

- The elaboration of strategies and guidance for the general population and for health care professionals, by the Project partners and by national "stakeholder panels" both in the contaminated territories of Belarus and in the three Member States. This will be done in the form of practical tools accessible to any interested person (i.e. handbook, simple computer program), taking account of different degrees of technical complexity, to provide general or more detailed information, and advice to follow in a contaminated territory in order to avoid unnecessary exposures in the course of day-to-day activities and to adopt a responsible and prudent attitude in regard to the protection of health.

The review of existing infrastructures aims at providing the European Commission and the involved Member States with a clear state of the art of the "readiness" of these countries to deal, in the longterm, with a contamination of the environment resulting from a nuclear accident or any other kind of contamination. An analysis of the potential of existing regulations and infrastructures to provide all concerned stakeholders in society with practical means to tackle the problems raised by living in a contaminated environment will be given. Gaps in national infrastructures will be highlighted as far as possible and recommendations made for new developments, particularly in view of the findings from the Belarusian experience, including ETHOS. 
As regards the elaboration of strategies and guidance for the general population and health care professionals, the key output is a handbook and complementary tools that contain comprehensive guidance for inclusive radiation monitoring, based on the findings from the ETHOS experience. These tools should provide the concerned inhabitants of a contaminated territory with the basic practical means to evaluate and directly participate in the management of the radiological situation. This handbook is to be accessible to the general population and the administrations, the local elected representatives and all concerned professionals but primarily the health professionals.

\section{THE STAKEHOLDER INVOLVEMENT PROCESS IN THE SAGE PROJECT}

According to the objectives of SAGE Project, stakeholders panels were set up during the first year of the project, in the four participating countries, i.e. Belarus, France, Germany and United Kingdom. Each of these national panels involved more than twelve participants, from a wide variety of professional fields: public health area (nurses, midwives, medical doctors), radiation protection experts, medical social workers, representatives from NGOs, teachers, elected representatives, nuclear authorities, experts in risk communication... Each national stakeholder panel met several times each year with the objective of elaborating the handbook and discussing in depth its form and contents.

The involvement of stakeholders in the project was on a voluntary basis. All participants expressed a personal commitment that was driven by the following:

- A personal interest to be involved in a project since its inception, and not only at the end for consulting or reviewing the outputs or decisions already taken by Project leaders

- $\quad$ The possibility to really affect and change the course of the Project (i.e. by directly influencing the objectives and approach, and on the way results and outputs can be exploited)

- The openness of the Project to accept new participants generally at the invitation of stakeholders already involved.

Beyond the development of the handbook (including editorial corrections and adaptation of its scope and content), the national stakeholders panels have also expressed their willingness to take forward specific issues.

In France, the stakeholder group is interested in making a strip cartoon that would deal with radioactivity and related problems which are tackled within SAGE, but more accessible to the general population and particularly young people. The general ideas and script contents have been discussed during stakeholders panels meetings. A preliminary project (detailed script and example illustrations) is to be prepared by a team constituted of some of the French stakeholder panel representatives, a professional cartoonist (who would further act as team co-ordinator) and a local association of young people interested in drawing art and in the production of various pedagogical tools. This preliminary project will be presented at the final SAGE workshop (beginning of 2005) to be eventually further sponsored.

In Germany and in Belarus, the stakeholder groups expressed the need to produce a version of the handbook and complementary computer tools in their native language, adapting also some of the contents to their national specific context. The UK stakeholders' are also keen to adapt the handbook for use in the UK and to broaden its scope to include other types of contaminant. A sub group of stakeholders has been set up to look specifically at the management of contaminated gardens and allotments used for domestic food production as well as options for restricting the gathering of free foods from the wild.

\section{TOOLS FOR AIDING INFORMATION AND DIALOGUE}

Different methodological concepts and practical tools have been developed to provide the local population and professionals, especially the health professionals, with practical means to better interpret and make use of the different kinds of radiological measurements at an individual or at a community level. Tools for aiding such information and dialogue about the problems posed by the radioactivity between the population and the professionals are intended to be accessible to any concerned person, independently of his/her background. 
The first tool is a handbook, which will be released as a loose-leaf modular format to facilitate revisions and updates, and also in an electronic "interactive" version, to be distributed via the Internet or CDs. The handbook contents have been developed using a series of questions likely to be posed by the population and the professionals. Four main entities have been considered: the household, the health professionals, the measurement professionals and the stakeholder advisory board.

The household is the place where concerns and questions regarding radioactivity and its effects on the health, especially the long-term effects on children, would arise and crystallise.

The health professionals include those responsible for the health surveillance and protection. They are not limited to the physicians but also include the staff of hospitals, nurses, pharmacists, psychologists, as well as school doctors and company doctors. The role of these persons would change due to the need to answer questions related to radioactivity. They would become the first point of contact for members of the public with regard to their concerns over their health and on the issues relating to radiological protection.

The measurement professionals are people in charge of measuring the contamination of foodstuffs and of the human body, and who are able to inform people about ambient dose rates at the local level. These functions are relatively new at the local level and need to be further defined. They should assure a service of measurement and advice for the local population, and for the whole society through the stakeholder advisory board.

The local stakeholder advisory board is composed of people with a wide range of skills at the local level (village, city quarter). It can rely on existing bodies or structures, or stand on its own. This body would collect data on the radiological situation from the different measurement sources (ambient dose rates, radiological quality of foodstuffs, whole body measurements). It would carry out statistical analyses of the data at local level, from which it will develop useful reference situations to help in the interpretation of the data by the various interested parties. Its work would not be limited to purely technical matters. It would work with the administrations (local, regional and national government) and with the interested professionals. It should involve representatives from the administrations, local elected representatives and non-governmental organisations to facilitate the exchange of information about the radiological situation for the whole society and hence improve the strategies for all interested parties.

The handbook contents are structured around the different concerns and questions of the aforementioned entities. Starting from these questions, practical "technical" sheets have been elaborated to provide information with differing degrees of complexity, according to the background knowledge of the reader. The practical sheets are centred around the following issues:

- $\quad$ Radioactivity: description, units, health effects, uptake in the body,

- Radiological measurements: ambient dose rates, foodstuffs contamination, body contamination,

- Exposure routes: external irradiation, ingestion and inhalation, transfer in the environment,

- Radiation monitoring: ambient dose rates, foodstuffs contamination, body contamination,

- Piloting tools: analysis of body contamination, analysis of ambient dose rates, comparison tools, data management,

- Improvement options: for the household, health professionals and stakeholder advisory board.

A computer program has been developed by CEPN to complement the handbook. It is based on uptake models for Man proposed by ICRP [7-8]. Starting from the results of whole body measurements, this computer program allows first estimates of the possible associated intakes in terms of total and mean daily ingested radioactivity. These estimates can then be further refined in an iterative process involving dialogue between the concerned person and the health professional or the measurement professional (see Figure 1). 


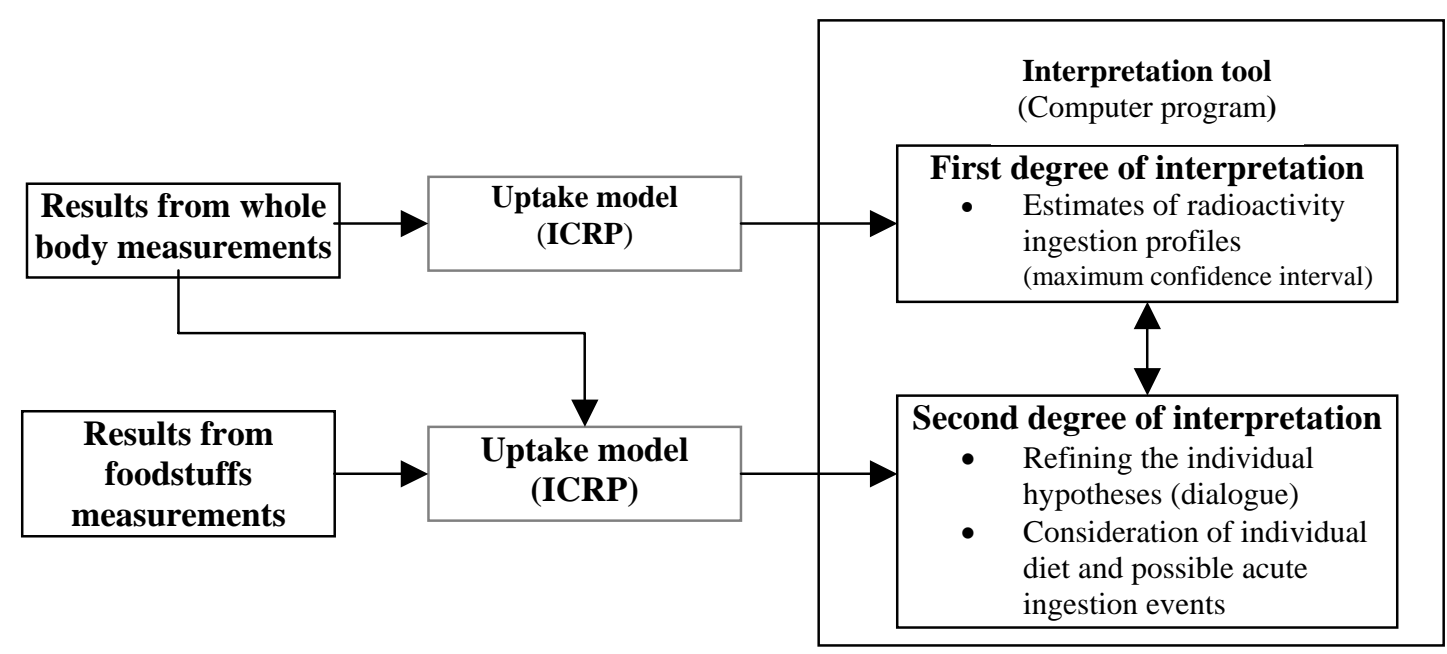

Figure 1. Principle scheme of the internal contamination and foodstuffs analysis computer program.

The main result expected from this tool is to facilitate the dialogue between concerned stakeholders and the general population, using quantitative information that they may contribute (partly and not as a unique criterion). It should then be possible to identify individual improvement options and to delineate local and national improvement strategies.

\section{CONCLUSION}

The SAGE Project provides an illustration of a participatory approach for the elaboration of methodological concepts and practical tools that contribute to the development of a "practical radiation protection culture” for people living in a long-term contaminated territory. The preliminary review of the existing infrastructures in France, Germany and the UK, has provided a clear state of the art of the "readiness" of these countries to deal, in the long-term, with a contamination of the environment resulting from a nuclear accident or any other kind of contamination. A handbook providing strategies and guidance for an inclusive system of radiation monitoring and protection is being developed, based on national stakeholder panels set up in the participating countries. The approach adopted in the SAGE Project has initiated a dialogue between different stakeholders, having varying expectations and concerns about the problems posed by radioactivity, but sharing a common willingness to tackle a problem which is not only a matter of experts. This Project has produced different tools that could further aid in facilitating this dialogue among a wider panel of stakeholders and that could help in the construction and dissemination of a practical radiation protection culture in Western Europe. A final workshop is scheduled for March 2005 to present the findings of the project to a wide audience and to investigate ways to implement the strategies and guidance on a practical level.

\section{References}

[1] The ETHOS Project in Belarus, Final Report, ETHOS(99)-RP(1), http://www.cepn.asso.fr/fr/ethos.html, (1999).

[2] Hériard Dubreuil G., et al., Chernobyl post-accident management: the ETHOS Project, Health Physics, Vol.77, pp.361-372, (1999). 
[3] Lepicard S., Hériard Dubreuil G., Practical improvement of the radiological quality of milk produced by peasant farmers in the territories of Belarus contaminated by the Chernobyl accident, Journal of Environmental Radioactivity, Vol. 56, № 1-2, pp. 241-253, (2001).

[4] Réhabilitation des conditions de vie dans les territories contaminés par l'accident de Tchernobyl: la contribution de l'approche ETHOS, Actes du Séminaire International de Stolyn, Biélorussie, 15-16 novembre 2001, http://www.cepn.asso.fr/fr/ethos.html.

[5] CORE Cooperation for Rehabilitation of living conditions in Chernobyl affected areas of Belarus Declaration of principles of the CORE Programme, Minsk, http://www.un.org/ha/chernobyl, (2003).

[6] http://www.ec-sage.net/

[7] Age-dependent doses to members of the public from intake of radionuclides: Part 1, Publication 56, International Commission on Radiological Protection, Annals of the ICRP, vol.20, No.2 (1989).

[8] Institut de Protection et de Sûreté Nucléaire, Le césium de l'environnement à l’homme, Collection IPSN, EDP Sciences, (2000). 